\title{
Partecipato è un aggettivo?
}

\section{Giuseppe Patota}

PUBBLICATO: 10 SETTEMBRE 2019

\section{Quesito:}

Alcuni utenti chiedono se sia corretto l'uso di partecipato (sul piano grammaticale, participio passato del verbo partecipare) in funzione di aggettivo, nel significato di 'condiviso' o 'con vasta affluenza di pubblico'.

\section{Partecipato è un aggettivo?}

I 1 fatto che la parola sia lemmatizzata (cioè classificata come voce autonoma) in molti vocabolari generali che danno conto dell'uso contemporaneo ci consente di rispondere di si. Valgano, fra le diverse adducibili, le testimonianze offerte dallo Zingarelli 2org e dal Devoto-Oli 20I8: il primo lemmatizza partecipato come participio passato di partecipare ma avverte che può essere adoperato anche come aggettivo nei significati, autonomi rispetto a quelli del verbo, di 'caratterizzato dalla partecipazione di più persone', di 'condiviso' (un'opinione partecipata da altri) e infine di 'che esprime intensa partecipazione' (fu un bacio lungo, partecipato, da un romanzo di Vasco Pratolini); il secondo lemmatizza partecipato senz'altro come aggettivo, ne dà il significato di 'condiviso', 'impostato su esperienze socialmente ampliate e individualmente approfondite' e allega, per illustrare quest'accezione, un esempio tratto dalla prosa giornalistica di Eugenio Scalfari: "il modo nuovo di produrre e di vivere, più creativo, più libero, più partecipato". Come si può vedere, c’è una perfetta sintonia fra i significati proposti da questi repertori e quelli proposti da ci ha rivolto le domande.

\section{Cita come:}

Giuseppe Patota, Partecipato è un aggettivo?, "Italiano digitale", 2019, X, 2019/3 (luglio-settembre) DOI: $10.35948 / 2532-9006 / 2020.3203$ 